This item was submitted to Loughborough's Research Repository by the author.

Items in Figshare are protected by copyright, with all rights reserved, unless otherwise indicated.

\title{
Developments in laser Doppler accelerometry (LDAc) and comparison with laser Doppler velocimetry
}

\section{PLEASE CITE THE PUBLISHED VERSION}

http://dx.doi.org/10.1016/S0143-8166(00)00002-6

\section{PUBLISHER}

(c) Elsevier

\section{VERSION}

AM (Accepted Manuscript)

\section{PUBLISHER STATEMENT}

This work is made available according to the conditions of the Creative Commons Attribution-NonCommercialNoDerivatives 4.0 International (CC BY-NC-ND 4.0) licence. Full details of this licence are available at: https://creativecommons.org/licenses/by-nc-nd/4.0/

\section{LICENCE}

CC BY-NC-ND 4.0

\section{REPOSITORY RECORD}

Rothberg, Steve, Alan Hocknell, and Jeremy M. Coupland. 2019. "Developments in Laser Doppler Accelerometry (Idac) and Comparison with Laser Doppler Velocimetry". figshare. https://hdl.handle.net/2134/19744. 


\title{
Developments in Laser Doppler Accelerometry (LDAc) and Comparison with Laser Doppler Velocimetry
}

\author{
Steve Rothberg, Alan Hocknell* and Jeremy Coupland \\ Department of Mechanical Engineering, Loughborough University, \\ Loughborough, Leicestershire, LE11 3TU, UK \\ *Now at Callaway Golf, 2285 Rutherford Road, Carlsbad, California 92009, USA
}

\begin{abstract}
This paper outlines the principles and early development of an interferometric technique for remote measurement of vibration acceleration - Laser Doppler Accelerometry (LDAc). One of the key advantages of LDAc over Laser Doppler Velocimetry (LDV) is its ability to measure extremely high vibration accelerations and shocks, effectively without limit, and this point is expanded upon in the paper. Early LDAc development showed how unwanted, velocity-dependent optical beats could occur on the photodetector but novel use of a frequency shifting device, whose primary purpose was for direction discrimination, was successful in isolating the required acceleration-dependent beat. A problem remained in the rate at which the velocity-dependent and acceleration-dependent beats broadened during target motion. In a further development, it was possible to 'select' a back reflection to produce a velocity-dependent beat that was NOT modulated in the presence of target motion. The acceleration-dependent beat could then be demodulated and preliminary results are given to demonstrate this outcome.
\end{abstract}




\section{INTRODUCTION}

Laser Doppler Velocimetry (LDV) is now an established technique for the measurement of vibration velocities. Hot, light or rotating targets are often quoted by commercial manufacturers as typical application areas and a whole range of new and improved products and processes owe their existence to the data obtained using LDV. In a basic LDV system, scattered light that has been Doppler shifted by the vibrating target is mixed with a coherent reference beam (often frequency-shifted for resolution of target direction) and heterodyned on a photodetector. This results in an optical beat at the detector at the difference frequency between target and reference beams. Frequency demodulation then produces a time-resolved analogue of the target vibration velocity.

A number of factors, however, have limited the versatility of LDV-based instruments and the first problem encountered is in maintaining the imbalance between target beam and reference beam paths to within the coherence length of the laser. This requires the Vibration Engineer, who may well lack any knowledge of laser technology, to control the target beam path, sometimes in situations where available access is already limited. To ease the requirement, lasers with long coherence length or even frequency-stabilised lasers can be used but this incurs extra expense and restricts choice of laser source. For example, advantage cannot be taken of new, visible diode lasers which are cheap and compact but have limited coherence length.

A second issue is that of the upper measurement limit. If the time dependent target velocity is $U(t)$ and the reference beam pre-shift is $f_{R}$, then the beat frequency, $f_{\text {beat }}$, will be: 


$$
f_{\text {beat }}=\left|f_{R}-\frac{2 U(t)}{\lambda}\right|
$$

where $\lambda$ is the laser wavelength. The laser wavelength and the bandwidth of the frequency demodulator are the only available controls over the sensitivity and working range of an LDV system. Currently available systems are limited to measurement of velocities in the range $1 \mu \mathrm{m} / \mathrm{s}$ to $10 \mathrm{~m} / \mathrm{s}$. With laser wavelength limited to the visible region for eye safety and no expectation of a step change in demodulator technology, there is no foreseeable opportunity to widen this range.

Advances in machinery and manufacturing technology have already passed the upper measurement limit. In a high performance internal combustion engine valve speeds of the order of $20 \mathrm{~m} / \mathrm{s}$ and valve bounce at potentially higher speeds are encountered ${ }^{1}$. In ultrasonically assisted manufacturing, surface accelerations up to $500,000 \mathrm{~g}$ at $20 \mathrm{kHz}$ (approximately $40 \mathrm{~m} / \mathrm{s}$ ) are predicted but this cannot yet be experimentally confirmed ${ }^{2}$. In pyroshock applications accelerations between 10,000g and 100,000g at frequencies between $10 \mathrm{~Hz}$ and $100 \mathrm{kHz}$ are reported ${ }^{3}$ - at the very limit of the best LDV systems.

In some applications, the vibration of interest occurs in the presence of significant whole body motion such as during impacts. In vehicle crashworthiness studies, whole vehicle velocities of the order of $10-20 \mathrm{~m} / \mathrm{s}$ are common but it is the localised deformation velocities which are of importance in analysis of energy dissipation ${ }^{4,5}$. Even the launch velocity of a golf ball can only be monitored for less than a third of the $450 \mu$ s duration of its impact with a golf club ${ }^{6}$. 
An increased upper measurable velocity limit would not necessarily overcome these problems because an instrument working range set to accommodate the whole body velocity component will result in poor sensitivity to the smaller fluctuating component of interest. It would be attractive to measure acceleration rather than velocity for such applications. Vibration Engineers are often interested in measuring acceleration because of its close relationship with force and because it is generally more acceptable to integrate to obtain velocity or displacement. LDV system outputs, which are often affected by speckle noise ${ }^{7}$, are not generally suitable for differentiation to obtain acceleration. At high velocities, the LDV system's inability to track the beat frequency would, in any case, preclude any attempt to obtain such an acceleration signal. A non-contact laser-based measurement of vibration acceleration has not been achieved previously and a prototype instrument capable of making this measurement is the subject of this paper.

\section{LASER DOPPLER ACCELEROMETRY (LDAc)}

\section{Translational Vibration Measurement}

The principle of Laser Doppler Accelerometry (LDAc) is most easily described by considering an application to measurement of translational acceleration ${ }^{8}$. In LDAc, the "reference" beam and the target beam are both taken from the target itself and the issue of maintaining path imbalance to within the coherence length of the laser now depends only on internal geometry. After being scattered from the target, the laser beam is amplitude divided and a path length imbalance introduced between each half of the beam as shown in figure 1 . Beams $\mathrm{A}$ and $\mathrm{B}$ also receive additional frequency shifts, $\Delta f_{A}$ and $\Delta f_{B}$, for direction 
discrimination. Recombination then produces an optical beat on the photodetector at frequency, $f_{\text {beat }}$, given by:

$$
f_{\text {beat }}=\left|\Delta f_{A}-\Delta f_{B}+\frac{2}{\lambda}[U(t)-U(t-\Delta l / c)]\right|
$$

where $c$ is the speed of light. If the target vibration velocity is represented as a Fourier integral then the velocity difference can be written and expanded as:

$$
[U(t)-U(t-\Delta l / c)]=\int_{0}^{\infty}[A(\omega) \sin (\omega t-\phi(\omega))-A(\omega) \sin (\omega(t-\Delta l / c)-\phi(\omega))] d \omega
$$

If an upper limit of $\omega_{\max }$ is defined such that the condition $\omega_{\max } \Delta l / c<<1$ holds then:

$$
f_{\text {beat }}=\left|\Delta f_{A}-\Delta f_{B}+\left(\frac{2 \Delta l}{c \lambda}\right)\left(\int_{0}^{\omega_{\max }}[\omega A(\omega) \cos (\omega t-\phi(\omega))] d \omega\right)\right|=\left|\Delta f_{A}-\Delta f_{B}+\left(\frac{2 \Delta l}{c \lambda}\right) \frac{\mathrm{d} U(t)}{\mathrm{d} t}\right|
$$

The beat frequency is therefore directly proportional to acceleration, offset from zero by the frequency shift $\left|\Delta f_{A}-\Delta f_{B}\right|$, provided $\omega_{\max } \Delta l / c<<1$.

Another optical velocity transducer - 'Velocity Interferometer System for Any Reflector' or $\operatorname{VISAR}^{9,10}$ - is capable of measuring velocities of several kilometres per second and was developed originally for the investigation of ballistic impacts. The VISAR geometry is also that of a long path imbalance interferometer, amplitude dividing light returning from the target into two components, one of which travels round an optical delay. The instrument 
counts interference fringes produced as a result of recombining the two components and target velocity is calculated. Velocity sensitivity adjustment is by appropriate choice of delay. Target motion direction is resolved by retarding the polarisation of light in the optical delay path such that two independent fringe patterns are formed in quadrature. For acceptable fringe visibility, however, a laser source with high temporal coherence is required. This would also be the case for the LDAc configuration shown in figure 1 in order to maintain sufficient sensitivity $(2 \Delta l / c \lambda)$ to accelerations in the range of general engineering interest, restricting application to very high accelerations, for example at elevated frequencies. This would still permit acceleration measurements that are currently impossible but it does not yet exploit the full potential of the instrument.

The measurement sensitivity can be maintained using a source with limited temporal coherence if the necessary path imbalance, $\Delta l$, is compensated by an equivalent imbalance elsewhere in the optical configuration. This very important development is shown in figure 2. The laser output is amplitude divided into two paths, labelled A and B, such that light on path A travels an additional imbalance, $\Delta l$, before recombining with light on path $\mathrm{B}$ to form a single beam incident on the target. Light backscattered from the target is amplitude divided again such that the light on path B now travels an additional imbalance, $\Delta l$. On recombination at the photodetector the path imbalance between A and B is negligible and the components of light are perfectly coherent. The preceding analysis of acceleration sensitivity remains valid and the application range is widened considerably through virtually unlimited choice of $\Delta l$. Furthermore, this well balanced internal optical geometry removes the requirement for the engineer to choose a target beam path length compatible with the coherence length of the laser and widens the choice of laser source to include even temporally quite incoherent sources such as an inexpensive red laser diode. 


\section{Comparison between LDAc and an LDV-based system with an electronic time delay}

The upper velocity limit of LDV instruments, set by the demodulator technology, has already been discussed. This section explores the possibility of producing a measurement of vibration acceleration by taking the photodetector output from an LDV configuration, electronically mixing (multiplying) this signal with an electronically time-delayed version of itself and demodulating the difference frequency component in the resulting output. From equation (1) this difference frequency, $f_{\text {diff }}$, can be written:

$$
f_{\text {diff }}=|| f_{R}-\frac{2 U(t)}{\lambda}|-| f_{R}-\frac{2 U(t-\tau)}{\lambda} \mid
$$

To facilitate comparison of equation (2) for LDAc and equation (5) for the LDV-based system, consider a scenario where $f_{R}=\left(\Delta f_{A}-\Delta f_{B}\right)=5 \mathrm{MHz}$, the vibration velocity is sinusoidal at angular frequency $\omega$ and $\omega \tau=\omega(\Delta l / c)=0.05$ rads. For the case where $f_{R} \geq(2 U / \lambda)_{\max }$, figure 3a shows the LDAc beat frequency (with its $5 \mathrm{MHz}$ offset removed) and figure 3b shows the LDV-based difference frequency. The LDV-based system's inability to discriminate direction is the obvious feature in the comparison but this could be remedied by electronically shifting one of the beats upwards in frequency. A further problem occurs when $(2 U / \lambda)_{\max }>f_{R}$, as shown in figure 4. In this case, the LDV-based difference frequency, shown in figure $4 \mathrm{~b}$, has a discontinuity enduring while the individual beat frequencies, $\left(f_{R}-\frac{2 U(t)}{\lambda}\right)$ and $\left(f_{R}-\frac{2 U(t-\tau)}{\lambda}\right)$, have different signs. The working range is therefore still capped by the magnitude of $f_{R}$. The LDAc beat frequency, shown in figure 4a, 
is not affected because it is dependent only on the velocity difference and not in any way on the absolute velocities. A further difficulty is introduced in tracking the required component in the LDV-based system because the maximum value taken by the difference frequency may be greater than the minimum frequency of the sum frequency component that also results from the mixing process.

This comparison confirms the unsuitability of an LDV-based velocity difference measurement and emphasises the unique potential of the LDAc configuration for measurements at high velocities.

\section{LDAc working range}

In a LDAc instrument, sensitivity is entirely adjustable through control of $\Delta l$ permitting application at low, moderate or high accelerations as required. A flexible instrument is envisaged in which working range can be "switched” by plug-in fibre-optic delay modules for the widest possible application range. Use of fibre-optics permits delays as long as several $\mathrm{km}$ to be used conveniently and easily.

With beat frequencies in the range $\left|\Delta f_{A}-\Delta f_{B} \pm(\Delta f)_{\max }\right|$ the maximum measurable acceleration, $a_{\max }$, is related to the path imbalance, $\Delta l$, by:

$$
a_{\max }=\frac{(\Delta f)_{\max } c \lambda}{2 \Delta l}
$$


Equation (6) shows that appropriate choice of $\Delta l$ allows measurement of as high an acceleration as is desired, with extreme values of $a_{\max }$ limited only by the practicalities of creating a very small or very large $\Delta l$. If $\omega_{\max } \Delta l / c \leq 0.1$ is set as the condition for linearity in equation (4), then the maximum vibration frequency at which measurements should be made, $f_{\max }$, is given by:

$$
f_{\max }=\frac{\omega_{\max }}{2 \pi}=\frac{0.1 c}{2 \pi \Delta l}
$$

Equation (7) shows that appropriate choice of $\Delta l$ allows measurement at as high a vibration frequency as is desired, limited only by the response of the frequency demodulator used.

Approximate working acceleration ranges and upper frequency limits for the LDAc configuration used in this study are shown in Table 1 for several path imbalances, $\Delta l$. These values are based on an instrument which uses a He-Ne laser $(\lambda=633 \mathrm{~nm})$, a demodulatable frequency range of $1.6 \mathrm{MHz} \pm 0.8 \mathrm{MHz}$ and a frequency demodulator with a dynamic range of approximately 100dB. For comparison, the measurement limits for piezo-electric accelerometers are around $1000 \mathrm{~km} / \mathrm{s}^{2}$ for shock and $100 \mathrm{~km} / \mathrm{s}^{2}$ for continuous vibration at frequencies up to $50 \mathrm{kHz}$ but access, mass-loading and attachment difficulties often prohibit full use ${ }^{11}$. Current frequency demodulator technology is also already capable of a tenfold increase in demodulatable frequency range compared to that used in this study. Converting acceleration to velocity, the maximum measurable velocity at $f_{\max }$ is approximately ten times the maximum measurable velocity in the equivalent velocimeter, increasing linearly with decreasing frequency to well beyond the velocity limit of current LDV systems. 
Further to this, if the maximum acceleration to be measured in a particular frequency range exceeds the maximum acceleration value shown in the table then a reduction in $\Delta l$ will accommodate the requirement, simultaneously increasing the upper frequency limit. It is an extremely important feature of the Laser Doppler Accelerometer that there is no upper measurement limit for vibration acceleration. The practical limit will only be encountered when the accuracy to which $\Delta l$ can be measured affects the measurement accuracy of the instrument. Until this very extreme limit is reached, reducing $\Delta l$ will allow measurement of higher and higher accelerations while maintaining dynamic range and frequency range.

\section{DEVELOPMENT OF THE OPTICAL CONFIGURATION}

\section{Coherence, polarisation and path imbalance}

The optical configuration shown in figure 2 is a useful aid in describing the principles of operation of LDAc but it is not a feasible practical arrangement. Light which travels faithfully along paths $\mathrm{A}$ and $\mathrm{B}$ in figure 2 produces an optical beat at a frequency proportional to the acceleration of the target surface when recombined on the photodetector. However, several other legitimate optical paths through the optical configuration exist. The longest path to the photodetector takes path A when travelling towards the target and path B on return from the target, whilst the shortest route to include the target is the reverse of this combination. In principle, the only beams that are coherent on the photodetector and thus able to produce a detectable beat are those that have travelled faithfully along paths A and B. In practice, however, unavoidable back reflections from optical surfaces and imperfect separation at beamsplitters direct a proportion of the total light to the photodetector by a number of undesirable paths. In particular when a path does not involve backscattering from the target, 
unwanted velocity-dependent optical beats occur on the photodetector. The polarisation state of the light can assist in minimising unwanted beats by removing many of the spurious beams while making maximum use of the available light but it cannot eliminate the velocitydependent beats.

A rotating diffraction grating is suited to the LDAc configuration because it provides the initial separation of light into paths A and B and simultaneously produces a frequency preshift of, in this case, $1.6 \mathrm{MHz}$. Despite poor light efficiency, associated with utilising only the first diffracted orders, the rotating diffraction grating was chosen for use in this study.

Initial investigation suggested the minimum practical path imbalance must be significantly greater than the coherence length of the laser source in order to reduce the intensity of velocity-dependent beats. With this in mind, an 80m optical fibre with refractive index of 1.5 provided a 120m path imbalance. Single mode polarisation preserving fibre was used in order to retain the polarisation information which distinguishes light on path A from that on path B. A schematic diagram of an LDAc configuration which incorporates an optical fibre is shown in figure 5. The required path imbalances on both paths A and B are implemented by light which passes in opposite directions along one axis of the same fibre in order to minimise any differential phase noise effects and equipment cost. The polarisation maintaining properties of the fibre are also used to rotate the polarisation of light propagating in the fibre by $90^{\circ}$, which simplifies the optical configuration slightly.

Analysis of the demodulated output showed that this LDAc configuration still exhibited sensitivity to the velocity of the target surface due to the combined effects of back reflections from optical surfaces and polarisation leakage at the beamsplitters. For example, light on path 
$\mathrm{B}$, which returns from the target through the fibre, interferes with the proportion of the light on path A which is unavoidably back reflected from the fibre entry, labelled (15) in figure 5. The two beams are co-linear with the same polarisation and, despite having travelled optical paths that differ by $120 \mathrm{~m}$, a strong $1.6 \mathrm{MHz}$ optical beat occurs on the photodetector. Since one of these beams is backscattered from the target and the other remains within the instrument, their associated beat is dependent on the velocity of the surface. The beams which have not travelled along the fibre or which are not back scattered from the target are of considerably greater intensity on the photodetector than those which have faithfully followed path A or B. One reason for this was the fibre launch/transmission efficiency which was around $50 \%$ for the input laser beam but only around $10 \%$ for light backscattered from the diffuse target. Consequently the unwanted, velocity-dependent beats dominate on the photodetector and are demodulated preferentially, emphasising the unreliability of coherence as a means to isolate the required beat.

\section{Frequency shift location}

Spurious optical beats on the photodetector caused by coherence between unwanted beams can be accommodated if the optical beat which carries the acceleration signal can be isolated. Novel use of the frequency-shifting device can distinguish the wanted and unwanted beats in addition to resolving target motion direction. The wanted and unwanted beats can be separated in frequency by careful positioning of the frequency-shifting device in the optical configuration. In the previous LDAc configuration, the majority of the unwanted light on the photodetector was the product of relatively intense back reflections from optical components close to the laser source. By locating the rotating diffraction grating close to the source, back reflected light was shifted in frequency by either $+800 \mathrm{kHz}$ or $-800 \mathrm{kHz}$ and was thus able to 
cause a beat at $1.6 \mathrm{MHz}$ with light backscattered from the target. A frequency-shifting device located further from the source leaves the most intense back reflections at the source frequency and thus able to produce only an $800 \mathrm{kHz}$ beat on combination with light backscattered from the target. Only light which has travelled faithfully along paths A and B will produce a $1.6 \mathrm{MHz}$ beat when recombined on the photodetector and this technique supersedes the unsuccessful use of coherence to isolate the acceleration-dependent beat.

In figure 6, the frequency shift is located well away from the laser source, such that light on path A travels through the fibre before reaching the rotating grating. Light on path A which is back reflected from the fibre entry is thus at the source frequency and unable to create a $1.6 \mathrm{MHz}$ beat with any other light in the instrument. Separation of the light on paths A and B is retained on beamsplitter (3) and through the grating, in order to ensure each path receives only one frequency shift. In this configuration, $50 \%$ of the light transmitted through the fibre on path A deviates along an unwanted path through components (12), (11) and (9) to the target and is not therefore frequency shifted by the rotating diffraction grating. This relatively intense, but unwanted, beam is directed back into the fibre after backscattering from the target but further attenuation and decreasing coherence with light on the main paths $\mathrm{A}$ and $\mathrm{B}$ diminish its importance. The main $800 \mathrm{kHz}$ (unwanted) beat in this optical configuration occurs between light returning from the target on path A and the back reflection from light at the fibre entry (16).

The photodetector output spectrum for a stationary target, figure 7a, shows the successful $800 \mathrm{kHz}$ separation of the velocity-dependent beat from the $1.6 \mathrm{MHz}$ beat, which carries the acceleration signal. The $800 \mathrm{kHz}$ beat is considerably stronger than the $1.6 \mathrm{MHz}$ beat because of the relatively intense back reflection from the fibre entry (16). This situation is unavoidable 
when using a single fibre to create both path imbalances since the photodetector must be located relatively close to (16) in order to gather the less intense wanted light, which returns from the target through the fibre on path B. In this fibre-coupled LDAc configuration the $800 \mathrm{kHz}$ and $1.6 \mathrm{MHz}$ signals are modulated by the velocity and acceleration of the target, respectively. For typical applications, however, broadening of the $800 \mathrm{kHz}$ peak occurs more readily as shown in figure $7 \mathrm{~b}$ and this will tend to mask the $1.6 \mathrm{MHz}$ signal in the Doppler spectrum, preventing its demodulation.

\section{Final configuration}

The optical configuration can be usefully adjusted to isolate the $800 \mathrm{kHz}$ beat by changing the polarisation arrangements to the optical configuration shown in figure 8. In this arrangement the light which is unavoidably diverted away from path A at beamsplitter (13) is not directed back into the fibre after backscattering from the target and is instead transmitted through beamsplitters (11) \& (19) and is incident upon the photodetector. It is useful to consider this light as a third legitimate optical path, C. Light on path $C$ is not frequency shifted by the rotating grating and a strong $800 \mathrm{kHz}$ beat is produced on the photodetector where light on path $\mathrm{C}$ is recombined with light on path $\mathrm{A}$, which has passed through the rotating diffraction grating and is thus shifted in frequency by $800 \mathrm{kHz}$. The beams which have followed paths A and $\mathrm{C}$ are both backscattered from the target, are of the same polarisation and exhibit maximum coherence as the path imbalance between them is very short.

Beams on main paths A and B leave beamsplitter (19) in orthogonal polarisation states and a linear polariser is positioned in front of the photodetector to allow an optical beat. The intensity of the light on path B is increased, at the expense of that on path A, by rotating the 
polarisation axis of the laser source such that an uneven separation of the light incident occurs through beamsplitter (2). This increases the intensity of the light returning from the target through the fibre, while simultaneously reducing the strength of the $800 \mathrm{kHz}$ beat between light on paths A and C.

The significance of this optical configuration is apparent in the presence of target motion. Both beams A and C are backscattered from the target and as such the $800 \mathrm{kHz}$ beat frequency is not modulated by target motion. The $1.6 \mathrm{MHz}$ signal is therefore sufficiently isolated in the Doppler signal spectrum to allow sidebands resulting from target oscillation to be studied. Tests using an ultrasonic cutting blade were conducted and sidebands observed around the $1.6 \mathrm{MHz}$ peak as shown in figure 9 . An independent measurement of the surface velocity confirmed that the extent of these sidebands represented sensitivity of this LDAc configuration to target acceleration

\section{CONCLUSIONS}

This paper has outlined the principles and early development of an interferometric technique for remote measurement of vibration acceleration - the most popular of the vibratory motion parameters. Compared to LDV, the optical design is more complex but this will be transparent to the user and the benefits are substantial. The LDAc principle is not only suited to use of an inexpensive laser source and associated components but it also simplifies use for the Vibration Engineer who may be unfamiliar with laser technology. This is achieved by the removal of the requirement to match optical paths to maintain coherence, which can be restrictive in LDV, by using an optical geometry in which path lengths are inherently matched regardless of the distance to the target. 
Early development has shown how back reflections in the geometry are responsible for creating unwanted optical beats on the photodetector even when optical path differences exceed the nominal coherence length of the laser. At the same time it proved impossible to use polarisation alone to separate the beams appropriately. A significant issue was that use of a single optical fibre to effect both path imbalances necessarily located the photodetector close to the most intense back reflection, from the optical fibre entry. This back reflected light was indistinguishable from the light returning through the fibre and the two beams were able to produce an unwanted optical beat. Additional complexity was introduced into the optical configuration in accommodating the polarisations necessary to direct the beams along the required paths whilst using only one axis of the optical fibre. This added optical complexity, exacerbated by use of a rotating diffraction grating as a frequency-shifting device, was wasteful of light and contributed to low signal intensity on the photodetector.

The acceleration-dependent (wanted) and the velocity-dependent (unwanted) optical beats can occur at the same mean frequency with the unwanted signal always the stronger, preventing demodulation of the acceleration signal. Frequency shifting at an appropriate point in the optical configuration was used successfully to separate the beats in frequency. However, modulation of the distinct beats in the presence of target motion broadened the velocity and acceleration signals, again causing difficulty in isolating the acceleration signal. It was possible, however, to 'select' a back reflection to produce a beat that is NOT modulated in the presence of target motion to be the main back reflection reaching the photodetector. The acceleration signal can then be demodulated and the unwanted beat frequencies can be filtered out 
This last effect points the way forward for future development. In a final instrument, application will be possible across a wide dynamic range and the technique's ability to "switch" working range will be unique among vibration transducers. The upper velocity limit of an LDV system is well understood. This paper has additionally shown the unsuitability of an LDV-based difference velocity measurement emphasising the unique nature of the LDAc ability to measure extremely high vibration accelerations and shocks, effectively without limit.

\section{ACKNOWLEDGEMENT}

The authors gratefully acknowledge the support of the Engineering and Physical Sciences Research Council during the period in which this work was carried out.

\section{REFERENCES}

1 A.H. Liu, T.C. Liu, M.J. Chen and T.C .Wang, "A comprehensive investigation of the high speed overhead-cam valve train dynamics in small internal combustion engine” Society of Automotive Engineers Technical Paper Series 951795. pp. 301-308 (1995).

2 M. Lucas and A.C. Smith, "Identification of Vibration Modes for the Redesign of Ultrasonic Plastic Welding Horns”, Proceedings of the Conference on Modern Practice in Stress and Vibration Analysis, Sheffield, UK, pp315-326, 1993.

3 C.J. Litz, "Laser Doppler Vibrometer, Unique use of Doe/Taguchi Methodologies in the Arena of Pyroshock (10 to 100,000Hz) Response Spectrum”, Proceedings of the First International Conference of Vibration Measurements by Laser Techniques: Advances and Applications, Ancona, Italy, pp432-449, 1994. 
4 J. Fenton, Handbook of vehicle design analysis. MEP, London, 1996.

5 N. Murray, When it comes to the crunch: the mechanics of car collisions, World Scientific Press, Singapore, 1994.

6 A. Hocknell, R. Jones and S.J. Rothberg, "Experimental Analysis of Impacts with Large Elastic Deformation: 1. Linear Motion”, Measurement Science and Technology, 7, pp12471254, (1996).

7 S.J. Rothberg, J.R. Baker and N.A. Halliwell, "Laser Vibrometry: Pseudo-Vibrations”, Journal of Sound and Vibration, 135(3), pp516-522 (1989).

8 S.J. Rothberg and J.M. Coupland, “The New Laser Doppler Accelerometer for Shock and Vibration Measurement”, Optics and Lasers in Engineering, 25, pp217-225, (1996).

9 L.M. Barker; R.E. Hollenbach, "Laser interferometer for measuring high velocities of any reflecting surface,” Journal of Applied Physics 43, pp4669-4675, (1972).

10 L.M. Barker, "Velocity interferometry for time-resolved high-velocity measurements," High-speed photography, videography and photonics. Proceedings of the SPIE 4277, pp116126, 1983.

11 M. Serridge and T. Licht, Piezo-Electric Accelerometers and Vibration Preamplifiers, Brüel and Kjær, Denmark, 1987. 


\section{FIGURES}

Figure 1: $\quad$ Schematic diagram showing the principle of a laser Doppler accelerometer.

Figure 2: $\quad$ Schematic diagram of a laser Doppler accelerometer incorporating path length compensation.

Figure 3: $\quad$ Frequency pre-shift greater than maximum target Doppler shift

(a) LDAc beat frequency.

(b) LDV-based system with electronic time delay.

Figure 4: $\quad$ Frequency pre-shift less than maximum target Doppler shift

(a) LDAc beat frequency.

(b) LDV-based system with electronic time delay.

Figure 5: $\quad$ Practical fibre coupled LDAc configuration using coherence and polarisation.

Figure 6: $\quad$ Practical fibre coupled LDAc configuration using frequency shift for beat discrimination.

Figure 7: $\quad$ Doppler signal spectra from the LDAc configuration in figure 6:

(a) stationary target (b) vibrating target.

Figure 8: $\quad$ Practical fibre coupled LDAc configuration for improved isolation of the acceleration-dependent beat.

Figure 9: Doppler signal spectrum from the LDAc configuration in figure 8 in the presence of target motion. 


\section{TABLE 1}

Frequency Ranges and Dynamic Ranges, as a function of path imbalance, for LDAc configuration used in the study.

\begin{tabular}{ccc}
\hline Path imbalance, $\Delta l$ & Acceleration range & $f_{\max }$ \\
\hline $5 \mathrm{~m}$ & $15000 \mathrm{~km} / \mathrm{s}^{2}$ to $150 \mathrm{~m} / \mathrm{s}^{2}$ & $1 \mathrm{MHz}$ \\
$50 \mathrm{~m}$ & $1500 \mathrm{~km} / \mathrm{s}^{2}$ to $15 \mathrm{~m} / \mathrm{s}^{2}$ & $100 \mathrm{kHz}$ \\
$500 \mathrm{~m}$ & $150 \mathrm{~km} / \mathrm{s}^{2}$ to $1.5 \mathrm{~m} / \mathrm{s}^{2}$ & $10 \mathrm{kHz}$ \\
\hline
\end{tabular}



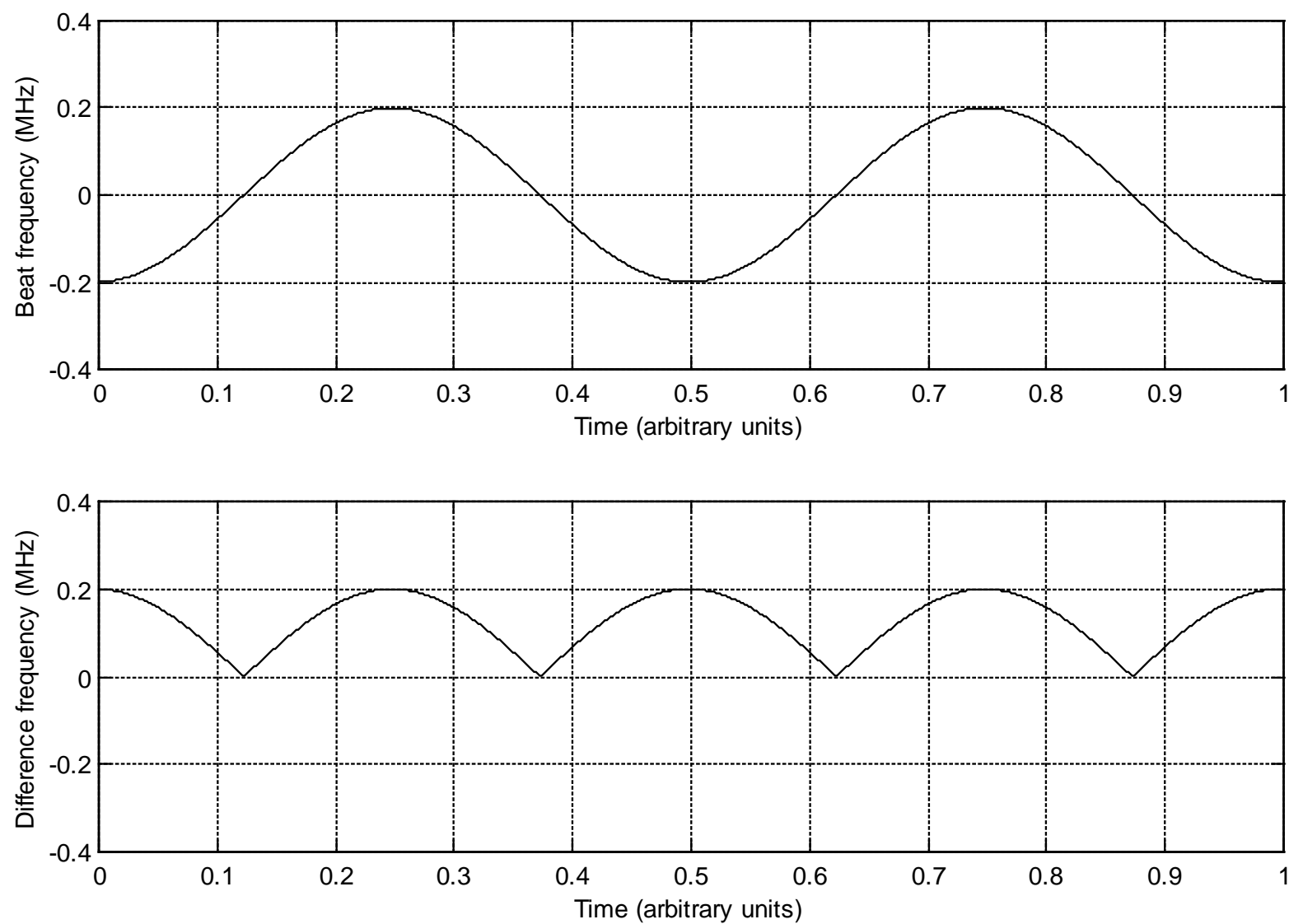

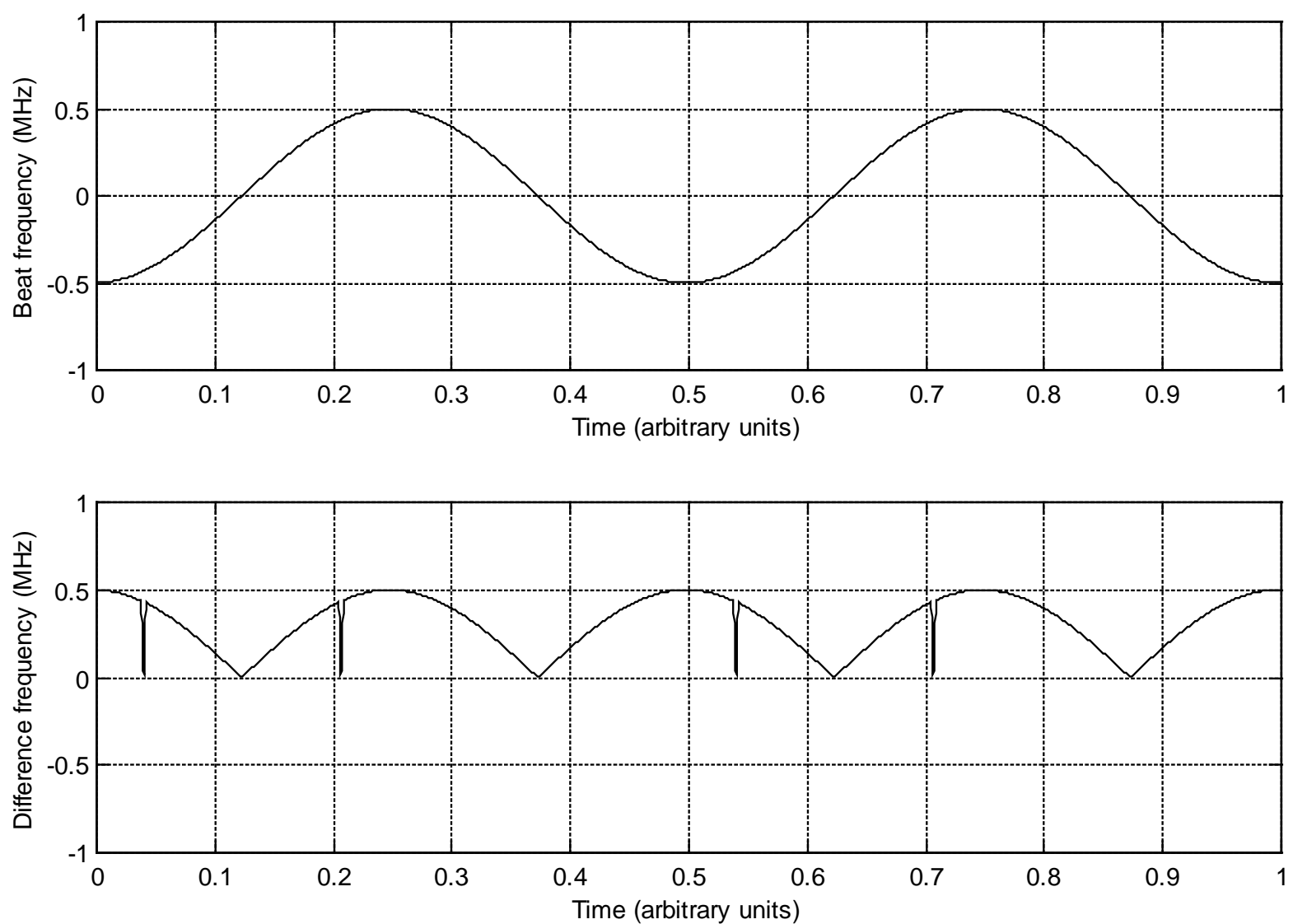